Forsch Komplementmed 18 | 1 | 11

Schweiz vergütet Komplementärmedizin in der obligatorischen Krankenversicherung

Die Anthroposophische Medizin, die Klassische Homöopathie, die Neuraltherapie, die Phytotherapie und die Traditionelle Chinesische Medizin (TCM) werden in der Schweiz ab 2012 über die obligatorische Krankenversicherung (Grundversicherung) vergütet. Diesen Entscheid fällte der Gesundheitsminister Didier Burkhalter am 13.01.2011. Die Aufnahme ist während 6 Jahren befristet. In dieser Zeit müssen die wissenschaftlichen Belege der Wirksamkeit, Zweckmässigkeit und Wirtschaftlichkeit der Methoden aktualisiert werden. Der Entscheid wurde aufgrund des Verfassungsartikels für Komplementärmedizin gefasst, welchem die Schweizer Stimmberechtigten am 17.06.2009 mit 67\% zugestimmt haben.

Der Dachverband Komplementärmedizin ist sehr erfreut über den Entscheid des Gesund- heitsministers, die Anthroposophische Medizin, Klassische Homöopathie, Neuraltherapie, Pflanzenheilkunde und TCM in die obligatorische Krankenversicherung (Grundversicherung) aufzunehmen. Die Auflagen - im wesentlichen die Aktualisierung der wissenschaftlichen Grundlagen - können erfüllt werden, so dass eine definitive Aufnahme wahrscheinlich ist. Der Gesundheitsminister wird die notwendigen Anstrengungen zur Konkretisierung des Verfassungsartikels gemäss seinen Aussagen in Zusammenarbeit mit der KomplementärmedizinBranche fortführen:

- Medizinalberufegesetz: Die Komplementärmedizin soll in die Ausbildungsziele der Studiengänge für Humanmedizin, Pharmazie, Zahnmedizin, Chiropraktik und Veterinärmedizin integriert werden.

- Heilmittelgesetz: Die Zulassung von komplementärmedizinischen und traditionellen Arzneimitteln soll erleichtert werden.

- Schaffung nationaler Diplome: Derzeit wer- den in Zusammenarbeit mit den Behörden zwei nationale Diplome für nichtärztliche Therapeuten ausgearbeitet.

- Förderung der Forschung: Die Komplementärmedizin erhält Zugang zu Forschungsmitteln, soweit die entsprechenden Beitragsgesuche den Erfordernissen der Forschung genügen.

- Schaffung von Instituten/Lehrstühlen für Komplementärmedizin an Universitäten: Die Schaffung von Hochschulinstituten oder von Lehrstühlen liegt in der Kompetenz der Kantone. Der Bund kann diese jedoch unterstützen, beispielsweise durch Gewährung von projektbezogenen Zuschüssen.

Weitere Informationen bei

Dachverband Komplementärmedizin DAKOMED c/o Köhler, Stüdeli \& Partner GmbH

Walter Stüdeli

Amthausgasse 18, 3011 Bern, SCHWEIZ

walter.stuedeli@dakomed.ch

\section{Expertenkonferenz}

Symptomatik von Gelenkerkrankungen verbessern

Mehr als 2 Millionen Patienten haben in Deutschland aufgrund von progressiven Gelenkerkrankungen ein künstliches Gelenk. Die medikamentöse Therapie bei diesen Erkrankungen ist nur symptomatisch und mit zahlreichen teilweise therapielimitierenden Nebenwirkungen behaftet. In der Langzeittherapie wird daher häufig auf adjuvante naturheilkundliche Präparate zurückgegriffen.

Um Wirksamkeit und Verträglichkeit einer in Deutschland neu eingeführten fixen Kombination aus Fischöl, Zink, Brennnessel-Blätter-Extrakt und Vitamin E (LIPOaktiv G) bei primärer Arthrose und rheumatoider Arthritis zu prüfen, diskutierten unter der Moderation des Pharmakologen Prof. Dr. Dr. Ulrich Borchard, Universität Düsseldorf, Experten aus Klinik und Praxis und formulierten ein Konsensuspapier.

Die fixe Kombination konnte in einer randomisierten, doppelblinden, Placebo-kontrollierten klinischen Studie eine überzeugende Wirksamkeit und Verträglichkeit dokumentieren [1]. Nach einer Therapiedauer von 3 Monaten konnte die Symptomatik der Osteoarthritis (Gelenkschmerzen, Morgensteifigkeit) signifikant verbessert werden $(p<0,001)$. Ein weiterer Vorteil ist das Potenzial, nicht-steroidale Antirheumatika (NSAR) sowie Analgetika einzusparen, so die Experten. Beispielsweise reduzierte sich in der LIPOaktiv-G-Studie im Laufe der Therapie der durchschnittliche Schmerzmittelverbrauch von 19,8 auf 6,5 Tabletten pro
Woche ganz erheblich. Hierin sahen auch die Experten eine ausgezeichnete Möglichkeit, die Verträglichkeit der chemisch-synthetischen Medikation wesentlich zu verbessern und damit Folgeerkrankungen wie Magenulkus und -blutungen zu vermeiden.

\section{Referenzen}

1 Jaquet A et al.: Phytalgic, a food supplement vs placebo in patients with osteoarthritis of the knee or hip: a randomized double-blind placebo-controlled clinical trial. Arthritis Res Ther 2010;12(1):105.

Weitere Informationen bei

Biomedica Pharma-Produkte GmbH

Magnolienweg 17, 63741 Aschaffenburg

info@lipoaktiv.de

www.lipoaktiv.de

\title{
Ticker+++ Ticker+++ Ticker+++ Ticker+++ Ticker+++ Ticker+++ Ticker+++
}

Solartium Dietetics GmbH. Die ergänzende bilanzierte Diät Aminotroph ${ }^{\circledR}$ ermöglicht - soweit bekann erstmals - eine diätetische Behandlung von Patienten mit Sarkopenie. Darunter versteht man «altersassoziierten Muskelschwund». Eine verbesserte Lebensqualität durch körperliche Fitness ist das Ziel. Der praktische Nutzen des neuen Lebensmittels wird durch allgemein anerkannte wissenschaftliche Daten gestützt. Erst kürzlich publizierte eine Forschergruppe um Professor Nisoli von der Universität Mailand eine Studie mit Aufsehen erregendem Ergebnis (http://www.ncbi.n/m.nih.gov/pubmed/20889128).

Solartium Dietetics $\mathrm{GmbH}$

Kathrin Weiss

kathrin.weiss@solartium.de
MEDICE Arzneimittel Pütter GmbH \& Co.KG. Es gibt einen Zusammenhang zwischen der Höhe des Homocysteinspiegels und dem Risiko, Jahre später an Demenz zu erkranken. Das zeigt erneut eine aktuelle Studie, in welcher der Zusammenhang zwischen B-Vitaminspiegel, Homocystein und Alzheimer-Demenz untersucht wurde. Ein ausgeglichener B-Vitaminstatus bietet demnach eine gute Chance, das Risiko einer Alzheimer-Demenz zu verringern. In Deutschland wurde für das B-Vitamin-Kombinationspräparat (Medyn ${ }^{\circledR}$ forte) in einer randomisierten, placebokontrollierten Studie nachgewiesen, dass es effektiv den Homocysteinspiegel senken kann.

Patzer PR GmbH

Dr. Maren Mundt

maren.mundt@patzer-pr.com
Kliniken Essen-Mitte. Der Lehrpreis der Medizinischen Fakultät der Universität Duisburg-Essen (Sommersemester 2010) ging an den Teil / das Seminar Naturheilkunde des Querschnittbereichs Rehabilitation, Physikalische Medizin und Naturheilverfahren, ausgerichtet von der Alfried Krupp von Bohlen und Halbach-Stiftungsprofessur für Naturheilkunde. Seit dem Wintersemester 2003/04 werden die Pflichtveranstaltungen der medizinischen Fakultät der Universität Duisburg-Essen in dem unabhängig durchgeführten Evaluationsverfahren EVALUNA von den Studenten bewertet.

Kliniken Essen-Mitte

Knappschafts-Krankenhaus

Prof. Gustav Dobos

gustav.dobos@uni-duisburg-essen.de

\section{KARGER} (ㅇ) 2011 S. Karger GmbH, Freiburg 